\title{
$R$-AUTOMORPHISMS OF $R[G]$ FOR $G$ ABELIAN TORSION-FREE
}

\author{
DAVID C. LANTZ
}

\begin{abstract}
Let $R$ be a commutative ring with identity and $G$ a torsion-free abelian group. This note describes for a reduced $R$ the group of $R$ automorphisms of the group ring $R[G]$ when either $R$ has finitely many idempotents or $G$ has finite torsion-free rank. It also describes the $R$ automorphisms of $R[G]$ for a general $R$ and $G$ finitely generated free.
\end{abstract}

Throughout this note, $R$ will denote a commutative ring with identity and $G$ will denote a torsion-free abelian group, written additively. Our purpose is to describe, under certain conditions, the ring automorphisms of the group ring $R[G]$ which fix each element of $R$, i.e., the $R$-automorphisms of $R[G]$. The question, of course, arises naturally, though our interest was aroused by Gilmer's work [G] on the $R$-automorphisms of $R[X]$, and sustained by the work on automorphisms of group algebras for finite groups, which has spanned decades (e.g., Peterson's work announced in recent issues of the Notices of the American Mathematical Society). It is somewhat surprising that the solution is quite simple in the case of a domain $R$, since Theorem 2 describes all $R$-automorphisms of $R\left[X, Y, Z, X^{-1}, Y^{-1}, Z^{-1}\right]$, while the description of all $R$-automorphisms of $R[X, Y, Z]$, even for $R$ an algebraically closed field, is an open problem of rather long standing.

We shall most of ten write an element of $R[G]$ as $\sum_{g \in G} a_{g} X^{g}$, where each $a_{g}$ is an element of $R$, zero except for finitely many $g$ in $G$; and we denote by $U(S)$ the group of units, i.e., invertible elements, in the ring $S$.

1. Proposition. Let $e_{1}, \ldots, e_{n}$ be a complete orthogonal family of nonzero idempotents in the ring $R$ (i.e., $\sum_{i} e_{i}=1, e_{i}^{2}=e_{i} \neq 0$, and if $i \neq j$, then $\left.e_{i} e_{j}=0\right)$, and for each $i$, let $\psi_{i}$ be a group automorphism of $G$ and $\phi_{i}$ a group homomorphism from $G$ to $U\left(e_{i} R\right)$. Then $X^{g} \rightarrow \Sigma_{i} \phi_{i}(g) X^{\psi_{i}(g)}$ determines an $R$-automorphism of $R[G]$.

Proof. Since the elements $X^{g}$ form a basis for the free $R$-module $R[G]$, the given correspondence does determine a $R$-endomorphism of $R[G]$ as an $R$-module. In showing that this endomorphism preserves multiplication, we note first that it suffices to assume that $n=1$, for the family $e_{1}, \ldots, e_{n}$ induces a ring direct sum decomposition of both $R$ and $R[G]$, and it suffices to verify the assertion on each summand. Accordingly we treat $e_{1}$ as 1 and drop subscripts on $\psi$ and $\phi$.

Received by the editors June 23, 1975.

AMS (MOS) subject classifications (1970). Primary 13B99, 20K15; Secondary $13 \mathrm{~B} 10$.

Key words and phrases. Group ring, automorphism, torsion-free rank. 
Consider elements $\sum_{g \in G} a_{g} X^{g}$ and $\sum_{g \in G} b_{g} X^{g}$ of $R[G]$. The product of their images is

$$
\begin{aligned}
\left(\sum_{g} a_{g} \phi(g) X^{\psi(g)}\right)\left(\sum_{g} b_{g} \phi(g) X^{\psi(g)}\right) & \\
& =\left(\sum_{g^{\prime}} a_{\psi^{-1}\left(g^{\prime}\right)} \phi\left(\psi^{-1}\left(g^{\prime}\right)\right) X^{g^{\prime}}\right)\left(\sum_{g^{\prime}} b_{\psi^{-1}\left(g^{\prime}\right)} \phi\left(\psi^{-1}\left(g^{\prime}\right)\right) X^{g^{\prime}}\right) \\
& =\sum_{g^{\prime}}\left(\sum_{g_{1}^{\prime}+g_{2}^{\prime}=g^{\prime}} a_{\psi^{-1}\left(g_{1}^{\prime}\right)}\right) \phi\left(\psi^{-1}\left(g_{1}^{\prime}\right) b_{\psi^{-1}\left(g_{2}^{\prime}\right)} \phi\left(\psi^{-1}\left(g_{2}^{\prime}\right)\right)\right) X^{g^{\prime}} \\
& =\sum_{g^{\prime}}\left(\sum_{g_{1}^{\prime}+g_{2}^{\prime}=g^{\prime}} a_{\psi^{-1}\left(g_{1}^{\prime}\right)} b_{\psi^{-1}\left(g_{2}^{\prime}\right)}\right) \phi\left(\psi^{-1}\left(g^{\prime}\right)\right) X^{g^{\prime}} \\
& =\sum_{g}\left(\sum_{g_{1}+g_{2}=g} a_{g_{1}} b_{g_{2}}\right) \phi(g) X^{\psi(g)}
\end{aligned}
$$

i.e., the image of their product.

Finally, the endomorphism under consideration has inverse determined by $X^{g} \rightarrow \phi\left(\psi^{-1}(g)\right)^{-1} X^{\psi^{-1}(g)}$, and so is an automorphism.

In certain cases, all $R$-automorphisms of $R[G]$ have either this form or a closely related one, as we see below. The notation Aut $_{R} R[G]$ will be used for the group of $R$-automorphisms of $R[G]$ and Aut $G$ for the group of automorphisms of $G$, both with the operation composition. The collection of group homomorphisms from $G$ to $U(R)$, denoted $\operatorname{Hom}(G, U(R))$, is a group under pointwise multiplication, and Aut $G$ acts on this group on the right by composition. So we can form the semidirect product

$$
\text { Aut } G \times \operatorname{Hom}(G, U(R)) \text {, }
$$

in which the operation is given by $\left(\psi_{1}, \phi_{1}\right)\left(\psi_{2}, \phi_{2}\right)=\left(\psi_{1} \psi_{2},\left(\phi_{1} \psi_{2}\right) \phi_{2}\right)$.

2. THEOREM. If $R$ is a domain, then

$$
\text { Aut }_{R} R[G] \cong \text { Aut } G \times \operatorname{Hom}(G, U(R)) \text {. }
$$

Proof. We have already seen that elements $\psi$ of Aut $G$ and $\phi$ of $\operatorname{Hom}(G, U(R))$ determine an $R$-automorphism of $R[G]$ by $X^{g} \rightarrow \phi(g) X^{\psi(g)}$. Conversely, an element $\Phi$ of Aut $_{R} R[G]$ must map each $X^{g}$, a unit, to a unit in $R[G]$, i.e., a unit in $R$ times $X^{g^{\prime}}$ for some element $g^{\prime}$ of $G$. Thus $\Phi$ induces functions $\psi: G \rightarrow G$ and $\phi: G \rightarrow U(R)$, and the fact that $\Phi$ preserves multiplication shows that $\psi, \phi$ are group homomorphisms. Finally, since $\Phi$ is an automorphism of the free $R$-module $R[G]$, it maps the basis $\left\{X^{g}\right\}$ bijectively onto a basis, so $\psi$ is bijective, i.e., an automorphism of $G$. Clearly the correspondence $\Phi \rightarrow(\psi, \phi)$ is the desired isomorphism.

The only application of the domain hypothesis in this proof was in the description of the units of $R[G]$, so it may be expected that the proof will generalize. For one example, we drop (fleetingly) the convention that $G$ is torsion-free: Higman $[\mathbf{H}]$ has shown that if $R$ is the ring of integers in an algebraic number field and $G$ is a finite abelian group, then the units of $R[G]$ of finite multiplicative order are precisely the monomials as in the proof of 
the theorem, and so we derive the same description of $\operatorname{Aut}_{R} R[G]$ in this case. For other applications of this idea, we first describe the units of $R[G]$ for a general $R$ and a torsion-free $G$.

3. Proposition. The units of $R[G]$ are precisely the elements of the form $\sum a_{g} X^{g}$ such that, for each prime ideal $P$ of $R$, exactly one $a_{g}$ is not in $P$.

Proof. If $\sum a_{g} X^{g}$ is a unit in $R[G]$, then for any prime $P$ in $R$, its image in $(R / P)[G](\cong R[G] / P[G])$ is a unit. But $R / P$ is a domain and $G$ is torsion-free, so this image has exactly one nonzero term; i.e., exactly one $a_{g}$ is not in $P$. Conversely suppose that $\sum a_{g} X^{g}$ satisfies the condition, and let $M$ be a maximal ideal of $R[G]$. Exactly one coefficient, say $a_{g_{0}}$, is not in the prime ideal $M \cap R$ of $R$, and $X^{g_{0}}$ is a unit, so $a_{g_{0}} X^{g_{0}}+\sum_{g \neq g_{0}} a_{g} X^{g}$, the sum of an element not in $M$ and one in $M$, is not in $M$. Since $M$ was an arbitrary maximal, $\sum a_{g} X^{g}$ is a unit.

4. Lemma. The nilradical of $R[G]$ is the set of elements $\sum a_{g} X^{g}$ all of whose coefficients $a_{g}$ are nilpotents in $R$.

Proof. Any prime ideal of $R[G]$ contains a prime of the form $P[G]$ where $P$ is prime in $R$, and the intersection of the $P[G]$ 's is clearly $N[G]$ where $N$ is the nilradical of $R$.

5. Corollary. For a unit $u$ in $R[G]$, there exist a complete set of orthogonal idempotents $e_{1}, \ldots, e_{n}$ and a nilpotent $t$ in $R[G]$ such that $u=t+\sum_{i=1}^{n} a_{i} X^{g_{i}}$ where $a_{i}$ is a unit in $e_{i} R$ for each $i$.

Proof. Since idempotents can be lifted modulo a nil ideal [L, p. 72, Proposition 1], it suffices to assume $R$ is reduced. If $a_{1}, \ldots, a_{n}$ are the nonzero coefficients of $u$, then for each maximal ideal $M$ of $R$, exactly one $a_{i}$ is not in $M$, so we can write $r_{1} a_{1}+\cdots+r_{n} a_{n}=1$; and if $i \neq j$, then $a_{i} a_{j}$ is in every prime ideal of $R$, so $a_{i} a_{j}=0$. It follows easily that $e_{i}=r_{i} a_{i}$ is idempotent, generates the same ideal as $a_{i}$, and satisfies $e_{i} e_{j}=0$ for $i \neq j$.

The next two results are stated for reduced rings. We shall consider the case of rings with nilpotents and finitely generated groups in Theorem 8 , but the proof in that case seems to depend heavily on the finite basis of the group, so that it cannot be applied here. Even the group structure of $\mathrm{Aut}_{R} R[G]$ is difficult to describe in that case. A weak finiteness assumption of some kind seems to be needed even in the reduced case, but the group structure of Aut $_{R} R[G]$ then admits a clear description.

6. THEOREM. Let $R$ be a reduced semiconnected ring, with minimal nonzero idempotents $e_{1}, \ldots, e_{n}$. Then $\mathrm{Aut}_{R} R[G]$ is isomorphic to the direct product of the groups Aut $G \times \operatorname{Hom}\left(G, U\left(e_{i} R\right)\right.$ ) (a semidirect product).

Proof. Since $e_{1}, \ldots, e_{n}$ is a complete orthogonal family, Proposition 1 shows that an element of the direct product determines an $R$-automorphism of $R[G]$. Conversely, given an $R$-automorphism $\Phi$ of $R[G]$, the restriction $\Phi_{i}$ of $\Phi$ to $e_{i} R[G]$ (which can be regarded as the composition of the inclusion $e_{i} R[G] \rightarrow R[G]$ with $\Phi$ and then with multiplication by $e_{i}$ ) is an $e_{i} R$-automorphism of $e_{i} R[G]$. Since 1 (i.e., $e_{i}$ ) and 0 are the only idempotents in the reduced ring $e_{i} R$, Corollary 5 and the fact that $\Phi_{i}\left(X^{g}\right)$ must be a unit show 
that $\Phi_{i}\left(X^{g}\right)$ is a monomial. The morphisms $\psi_{i} \in$ Aut $G$ and $\phi_{i} \in$ $\operatorname{Hom}\left(G, U\left(e_{i} R\right)\right)$ arise as in the domain case, and $\Phi\left(X^{g}\right)=\Sigma_{i} \Phi_{i}\left(X^{g}\right)=$ $\sum_{i} \phi_{i}(g) X^{\psi_{i}(g)}$. It is easily seen that the group structure on $\operatorname{Aut}_{R} R[G]$ is as claimed.

7. THEOREM. For a reduced ring $R$ and a (torsion-free abelian) group $G$ of finite torsion-free rank, $\mathrm{Aut}_{R} R[G]$ is the direct limit of the directed system of groups described as follows: For each complete orthogonal family of nonzero idempotents $e_{1}, \ldots, e_{n}$, form the direct product of the groups

$$
\text { Aut } G \times \operatorname{Hom}\left(G, U\left(e_{i} R\right)\right)
$$

(a semidirect product); and if $e_{1}^{\prime}, \ldots, e_{m}^{\prime}$ is another such family which "refines" $e_{1}, \ldots, e_{n}$ (i.e., for which there is a partition $S_{1}, \ldots, S_{n}$ of $\{1, \ldots, m\}$ such that $e_{i}=\sum_{j \in S_{i}} e_{j}^{\prime}$ for each $i$ ), then the map

$$
\prod_{i}\left(\text { Aut } G \times \operatorname{Hom}\left(G, U\left(e_{i} R\right)\right)\right) \rightarrow \prod_{j}\left(\operatorname{Aut} G \times \operatorname{Hom}\left(G, U\left(e_{j}^{\prime} R\right)\right)\right)
$$

is defined by mapping Aut $G \times \operatorname{Hom}\left(G, U\left(e_{i} R\right)\right)$ to

$$
\prod_{j \in S_{i}}\left(\text { Aut } G \times \operatorname{Hom}\left(G, U\left(e_{j} R\right)\right)\right)
$$

$\operatorname{via}(\psi, \phi) \rightarrow\left(\left(\psi, e_{i}^{\prime} \phi\right)\right)_{j}$.

Remark. Since each of these maps is injective, Aut $_{R} R[G]$ may be regarded as the directed union of these groups.

Proof. By Proposition 1, each element of $\Pi_{i}\left(\right.$ Aut $\left.G \times \operatorname{Hom}\left(G, U\left(e_{i} R\right)\right)\right)$ gives rise to an element of $\mathrm{Aut}_{R} R[G]$. Conversely suppose $\Phi \in \mathrm{Aut}_{R} R[G]$. Let $b_{1}, \ldots, b_{m}$ be a basis for a free subgroup $F$ of $G$ such that $G / F$ is torsion. For each $j=1, \ldots, m$, there is a complete orthogonal family of nonzero idempotents $e_{j, 1}, \ldots, e_{j, n_{j}}$ such that $e_{j, i} \Phi\left(X^{b_{j}}\right)$ has only one nonzero term. If we take a common refinement $e_{1}, \ldots, e_{n}$ of these families, then $e_{i} \Phi\left(X^{b_{j}}\right)$ has only one nonzero term for each $i, j$. Let $g \in G$ and write $k g=\Sigma_{j} z_{j} b_{j}$ where $k, z_{j}$ are integers and $k \neq 0$; then $e_{i} \Phi\left(X^{g}\right)^{k}=\prod_{j} e_{i} \Phi\left(X^{b_{j}}\right)^{z_{j}}$ has only one nonzero term. Since $G$ is torsion-free (so that it can be totally ordered) and $R$ is reduced, it follows that $e_{i} \Phi\left(X^{g}\right)$ has only one nonzero term. Now the restriction of $\Phi$ to $e_{i} R[G]$ is an $e_{i} R$-automorphism of $e_{i} R[G]$ with the property that the image of $X^{g}$ (or $e_{i} X^{g}$ ) is a monomial for each $g$ in $G$. The description of $\psi_{i}$ and $\phi_{i}$ follow as in the domain case.

Since a finitely generated torsion-free abelian group is free, the hypotheses on $G$ for a general $R$ (not necessarily reduced) are quite restrictive. But the ideas (drawn directly from $[\mathbf{G}]$ and $[\mathbf{B R}]$ ) which are used to show the induced endomorphism is bijective seem to require finite generation. Also, although our elementwise description of $\mathrm{Aut}_{R} R[G]$ determines the composition operation, its formal specification is sufficiently forbidding and uninformative that it seems wiser to omit it.

8. THEOREM. The R-automorphisms of $R\left[\mathbf{Z}^{n}\right]$ are determined as follows: Let $b_{1}, \ldots, b_{n}$ be the natural basis for $\mathbf{Z}^{n}$ and for each $i=1, \ldots, n$, let $t_{i}$ be nilpotent in $R\left[\mathbf{Z}^{n}\right]$. Let $e_{1}, \ldots, e_{m}$ be a complete orthogonal family of nonzero idempotents, and let $u_{i, j} \in R, b_{i, j} \in \mathbf{Z}^{n}$ satisfy: 
(1) $b_{1, j}, \ldots, b_{n, j}$ is a basis for $\mathbf{Z}^{n}$ for each $j=1, \ldots, m$, and

(2) $u_{i, j}$ is a unit in $e_{j} R$ for each $i$ and $j$.

Then the correspondence $X^{b_{i}} \rightarrow t_{i}+\sum_{j} u_{i, j} X^{b_{i j}}, i=1, \ldots, n$, determines an $R$-automorphism of $R\left[\mathbf{Z}^{n}\right]$. All $R$-automorphisms of $R\left[\mathbf{Z}^{n}\right]$ are so determined.

Proof. Given an $R$-automorphism of $R\left[\mathbf{Z}^{n}\right]$, the image of $X^{b_{i}}$ must be a unit, so we must have a complete orthogonal family of nonzero idempotents $e_{i, 1}, \ldots, e_{i, m_{i}}$ and elements $v_{i, j} \in R, t_{i} \in R\left[\mathbf{Z}^{n}\right], b_{i, j} \in \mathbf{Z}^{n}$ such that $v_{i, j}$ is a unit in $e_{i, j} R, t_{i}$ is nilpotent, and $X^{b_{i}} \rightarrow t_{i}+\sum_{j} v_{i, j} X^{b_{i j}}$. Let $e_{1}, \ldots, e_{m}$ be a common refinement of the families $\left\{e_{i, 1}, \ldots, e_{i, m_{i}}\right\}_{i}$ and set $u_{i, j}=e_{j} v_{i, j^{\prime}}$ whenever $e_{j} e_{i, j^{\prime}} \neq 0 \quad\left(j=1, \ldots, m ; j^{\prime}=1, \ldots, m_{i}\right)$. Then $X^{b_{i}} \rightarrow t_{i}+\sum_{j} u_{i, j} X^{b_{i j}}$. We must still show that, for fixed $j,\left\{b_{1, j}, \ldots, b_{n, j}\right\}$ is a basis for $\mathbf{Z}^{n}$, and for this we may assume that $R$ is reduced and, after multiplying by $e_{j}$, that the automorphism is given by $X^{b_{i}} \rightarrow u_{i} X^{b_{i j}}$, where $u_{i}$ is a unit. It follows that the image of any $X^{g}, g \in \mathbf{Z}^{n}$, is a monomial, so again the automorphism is given by $X^{g} \rightarrow \phi(g) X^{\psi(g)}$ where $\psi \in$ Aut $G$ and $\phi \in \operatorname{Hom}(G, U(R))$. Since $\psi$ is an automorphism it takes the basis $\left\{b_{i}\right\}_{i}$ bijectively to the basis $\left\{\psi\left(b_{i}\right)=b_{i, j}\right\}_{i}$.

Conversely, suppose $e_{1}, \ldots, e_{m}$ is a complete orthogonal family of nonzero idempotents, $t_{1}, \ldots, t_{n}$ are nilpotents in $R\left[\mathbf{Z}^{n}\right]$, and $u_{i, j} \in R, b_{i, j} \in \mathbf{Z}^{n}$ satisfy (1) and (2). The mapping of the polynomial ring $R\left[X^{b_{1}}, \ldots, X^{b_{n}}\right]$ to $R\left[\mathbf{Z}^{n}\right]$ determined by $X^{b_{i}} \rightarrow t_{i}+\sum_{j} u_{i, j} X^{b_{i j}}$ takes each $X^{b_{i}}$ to a unit, so it has a unique extension to an $R$-algebra homomorphism

$$
R\left[\mathbf{Z}^{n}\right]=R\left[X^{b_{1}}, \ldots, X^{b_{n}}\right]_{\left(X^{b_{1}} \ldots X^{b_{n}}\right)} \rightarrow R\left[\mathbf{Z}^{n}\right] .
$$

We must show this endomorphism is an automorphism, and for this we may multiply by $e_{j}$ and assume $m=1$.

To see that it is surjective, we show that each $X^{ \pm b_{i}}$ is in its image. The first step is to write $b_{i}=\sum_{j} k_{i, j} b_{j, 1}$, where $k_{i, j} \in \mathbf{Z}$, and note that

$$
X^{b_{i}}+t_{i}^{\prime}=\prod_{j}\left(u_{j, 1}^{-1}\left(t_{j}+u_{j, 1} X^{b_{j, 1}}\right)\right)^{k_{i, j}}
$$

is in this image as is its inverse $X^{-b_{i}}+t_{n+i}^{\prime}$; here each $t_{i}^{\prime}, i=1, \ldots, 2 n$, is nilpotent. We now induct on the index of nilpotence of the ideal of $R$ generated by the coefficients of $t_{1}^{\prime}, \ldots, t_{2 n}^{\prime}$ to show that $X^{ \pm b_{i}}$ is in $R\left[X^{b_{1}}+t_{1}^{\prime}, \ldots, X^{-b_{n}}+t_{2 n}^{\prime}\right]$ (and hence is in the image): If this index is 1 , then each $t_{i}^{\prime}$ is 0 and we are finished. Assume this index is at least 2 and write $t_{i}^{\prime}=\sum_{j} a_{j} X^{g_{j}}$, where $g_{j}=\sum_{k} z_{j, k} b_{k}, z_{j, k} \in \mathbf{Z}$, and $a_{j} \in R$ is nilpotent. Consider

$$
X^{b_{i}}+t_{i}^{\prime}-\sum_{j} a_{j} \prod_{k}\left(X^{b_{k}}+t_{k}^{\prime}\right)^{z_{j, k}}=X^{b_{i}}+t_{i}^{\prime \prime},
$$

where $t_{i}^{\prime \prime}$ is a nilpotent whose coefficients all lie in the square of the ideal generated by the coefficients of $t_{1}^{\prime}, \ldots, t_{2 n}^{\prime}$. Hence if we do this for each $i$, $i=1, \ldots, n$, and define $t_{i+n}^{n}$ by $X^{-b_{i}}+t_{i+n}^{\prime \prime}=\left(X^{b_{i}}+t_{i}^{\prime \prime}\right)^{-1}$, then the index of nilpotence of the ideal generated by the coefficients of $t_{i}^{\prime \prime}, \ldots, t_{2 n}^{\prime \prime}$ is strictly smaller than that for $t_{1}^{\prime}, \ldots, t_{2 n}^{\prime}$, so

$$
X^{ \pm b_{i}} \in R\left[X^{b_{1}}+t_{1}^{\prime \prime}, \ldots, X^{-b_{n}}+t_{2 n}^{\prime \prime}\right] \subseteq R\left[X^{b_{1}}+t_{1}^{\prime}, \ldots, X^{-b_{n}}+t_{2 n}^{\prime}\right] .
$$

It follows from the above that the endomorphism is an automorphism, for 
we can construct an inverse: Form the ring $R_{0}$ by adjoining to the prime subring of $R$ the coefficients of each $u_{i, 1} X^{b_{i, 1}}+t_{i}$ and its inverse, and finitely many other elements (namely $u_{i, 1}^{-1}$ for each $i$ ) so that the restriction of the endomorphism to $R_{0}\left[\mathbf{Z}^{n}\right]$ is onto. As a surjective endomorphism of the Noetherian ring $R_{0}\left[\mathbf{Z}^{n}\right]$, this restriction is an automorphism, and since the inverse fixes $R_{0}$ pointwise it has a unique extension to an $R$-endomorphism of $R\left[\mathbf{Z}^{n}\right]$. The compositions in both directions of our first endomorphism with this extended one fix each $X^{g}$ and each element of $R$, so both compositions are the identity.

ACKNOWLEDGEMENT. The author would like to thank his dissertation advisor, Professor James W. Brewer, for suggesting the problem and resources on it.

\section{REFERENCES}

[BR] J. W. Brewer and E. A. Rutter, Isomorphic polynomial rings, Arch. Math. (Basel) 23(1972), 484-488. MR 47 \#8609.

[G] R. W. Gilmer, Jr., R-automorphisms of $R[X]$, Proc. London Math. Soc. (3)18(1968), 328-336. MR 37 \#207.

[H] G. Higman, The units of group-rings, Proc. London Math. Soc. (2)46(1940), 231-248. MR 2 , 5.

[L] J. Lambek, Lectures on rings and modules, Blaisdell, Waltham, Mass., 1966. MR 34 \#5857.

Department of Mathematics, University of Kansas, Lawrence, Kansas 66044

Current address: Department of Mathematics and Computer Science, University of Nebraska, Omaha, Nebraska 68101 